\title{
HIGH ENERGY PROCESSES IN THE INTERSTELLAR MEDIUM
}

\author{
Catherine J. CESARSKY \\ Service d'Astrophysique/DAPNIA \\ CE SACLAY, 91191 Gif sur Yvette Cedex, France
}

\begin{abstract}
The relationship between high energy phenomena and the interstellar medium is wide. I had to make a selection, and only cover a few aspects : cosmic rays, gamma rays, correlation between radio synchrotron and infra red emission and its influence on cosmic rays ; and finally some new observational results.
\end{abstract}

Cosmic rays are a very important component of the interstellar medium, since their energy content is comparable to that of the gas and the magnetic field ; thus, they "belong" in a meeting such as this. The general shape of the energy spectrum - as a power law of index 2.7 - has been known for a long time ; in 1954, Fermi suggested that cosmic rays are accelerated by their random collisions with interstellar clouds, thus accounting for the spectrum shape, but he could not predict the value of the power index. At present, we know, more precisely, that the spectrum of cosmic ray protons between $10 \mathrm{GeV}$ and $10^{6} \mathrm{GeV}$ is a very straight, essentially featureless power law in $\mathrm{E}^{-}$ 2.7. Newer data from the JACEE experiment (Burnett et al. 1990) agree very well with results known before for protons. They found a surprising result for He, not explained at the moment ; for the other species, the results follow quite well those obtained at lower energy.

The cosmic ray anisotropy is very low ; at energies above $=10^{14} \mathrm{eV}$ it increases with energy like $\mathrm{E}^{0.5}$, but at lower energy it appears to remain roughly constant at $<0.1 \%$. This very small anisotropy indicates that cosmic rays are very well confined, otherwise we would see their sources in their angular distribution. Why are they so well confined ? One possibility, if the galactic magnetic field is closed, is that cosmic rays are trapped for ever in the galaxy, where they slowly loose their energy through collisions. In that case, the "grammage", or amount of matter traversed by $1 \mathrm{GeV}$ cosmic rays, would be $=100 \mathrm{~g} / \mathrm{cm}^{2}$. Now, it may be that the galactic magnetic field is closed, but that cosmic rays inflate bubbles in it, and finally puncture them and escape in the halo (Parker, 1969). This gives rise to the "leaky-box" model of cosmic ray confinement, where cosmic rays are very well confined to a disk or a thick disk, and once in a while encounter a hole and escape. The possibility most favoured by cosmic ray specialists is that cosmic rays diffuse in the interstellar medium, either because of interactions with large scale magnetic field irregularities, or because of resonant scattering with hydromagnetic waves of wavelength comparable to their Larmor radius. In addition, the magnetic field lines make a random walk in the disk, and sometimes they get close to the border and cosmic rays can escape. 
Much of our additional inferences on cosmic ray behaviour stems from our knowledge of the elemental composition. Some elements, such as $\mathrm{Li}, \mathrm{Be}, \mathrm{B}, \mathrm{Sc}$ and $\mathrm{Va}$, which are very rare in solar matter, are relatively abundant in cosmic rays. The reason of this difference is well known : as cosmic rays travel in interstellar space, they undergo collisions with interstellar nuclei, giving rise to spallation reactions, so that heavy nuclei break up into lighter ones. We distinguish between "primary" elements, such as $\mathrm{C}$ or $\mathrm{O}$, which were present at the source, and secondary elements, such as $\mathrm{Li}, \mathrm{Be}, \mathrm{B}$, created by spallation reactions in the interstellar medium. The abundances of the secondary elements are a measure of the amount of material the cosmic rays have traversed.

In the leaky-box model, for each species the observed density $\mathrm{Ni}$, the source density $\mathrm{Q}_{\mathrm{i}}$ and the grammage traversed before escape, $\lambda \mathrm{e}$, are related through :

$$
N_{i}=\frac{Q_{i} / \rho c+\sum_{j>i} N_{j} \sigma_{j i} / m}{\sigma_{i} / m+1 / \lambda_{e}}(1)
$$

where $\sigma_{i j}$ is the spallation cross section from specie $j$ to specie $i, \sigma_{i}$ the destruction cross section of specie $i$, and $m$ the proton mass.

We know that galactic cosmic rays have a large scale height, much greater than that of the gas. Thus a reasonable diffusion model will feature a narrow disk, containing most cosmic ray sources, and a wide cosmic ray confinement region, thick disk or halo. In this case, it can be shown that the abundances of the elements are still described by eq.

(1), where $\lambda_{e}$ is related to the diffusion coefficient $D$ :

$$
\mathrm{D}=\left(\mathrm{n}_{\mathrm{o}} \cdot \mathrm{hH} \mathrm{m} \mathrm{c}\right) / \lambda_{\mathrm{e}}
$$

where $n_{o}$ is the gas number density in the disk, $h$ the gas scale height, and $H$ the cosmic ray scale height. (Ginzburg et al. 1980). Thus, the simple equation (1) can encompass many physical situations.

To study cosmic ray propagation, we can apply equation (1) at various energies or rigidities ( $R=p c / e Z$, measured in Giga Volts, is proportional to the cosmic ray momentum $\mathrm{p}$ and to its gyration radius ). At rigidity $<5 \mathrm{GV}$, the escape length appears

to be constant or proportional to $\mathrm{v} / \mathrm{c}$ and $\approx 10 \mathrm{~g} / \mathrm{cm}^{-2}$, but at higher rigidity at least up to $=50 \mathrm{GV}$, it is proportional to $\mathrm{R}^{-0.6}$ (Engelmann et al. 1990 and reference therein). The first thing we notice is that the mean grammage of cosmic rays is much less than the $100 \mathrm{~g} / \mathrm{cm}^{2}$ expected if they are trapped for ever in the galaxy; thus, they do escape. The fact that at least in the rigidity range 5-50GV, the ratio of the abundances of secondary to primary elements decreases when the energy increases has been known for almost twenty years ; it is interpreted as implying that the escape length is energy dependent, so that cosmic rays of higher energy escape more readily from the galaxy.

In the framework of a diffusion model with energy independent escape boundaries, it implies that the diffusion coefficient $\mathrm{D} \alpha \mathrm{R}+0.6$ Then, the spectrum of cosmic rays which we observe is not that of the source ; correcting the observed spectrum for propagation effects, we find a source spectrum $\alpha \mathrm{R}^{2.1}$. 
For the past fourteen years, the favoured explanation of this spectral shape is that cosmic rays are accelerated in the vicinity of supernova shocks (e.g. see review by Blandford and Eichler, 1987). Energetic arguments indicate that the supernova phenomenon is related to cosmic ray origin. From the observed energy density and escape grammage of cosmic rays, we can derive an energy requirement for galactic cosmic rays; we indeed find that if supernova shocks can transmit $10 \%$ of their energy to cosmic ray, they can be the accelerating agent. How is the energy transmitted? Cosmic rays do not "see" the supernova shock, which is very thin compared to a cosmic ray gyration radius, but every time they cross it, in one or the other direction, they increase their energy by a factor $=\left(1+v_{S} / c\right)$, where $v_{S}$ is the shock velocity. Since they are trapped by small scale turbulence in the vicinity of the shock, they cross it many times, getting substantial acceleration. It is possible to show that the spectrum of the accelerated particles depends only on the ratio of the densities on both sides of the shock ; in particular, for a very strong shock, this ratio is equal to 4 , and the spectrum is proportional to $\mathrm{p}^{-2}$.

Much work has been done on this acceleration mechanism, which has had some success in accounting for the acceleration of galactic cosmic rays, but a number of problems remain. The mechanism, based on a diffusion process, is slow, while supernova shocks evolve fast. Even under the most optimistic assumptions about shock evolution, the maximum energy attained cannot be higher than $10^{14} \mathrm{eV}\left(\mathrm{B} / 10^{-6} \mathrm{G}\right)$ for protons. (Lagage and Cesarsky 1983). If a more realistic account is taken of the space distribution of the small scale turbulence at various times, the limit is decreased to $2.10^{12} \mathrm{eV} \times\left(\mathrm{B} / 10^{-6} \mathrm{G}\right)$. But we recall that there is no feature in the proton spectrum in this energy range up to $\approx 10^{15} \mathrm{eV}$. To reach $10^{15} \mathrm{eV}$, it is necessary to invoke fields much stronger than the general interstellar medium field. Völk and Biermann (1988) propose that cosmic rays are accelerated by a supernova shock which is still traversing a bubble created by winds ejected by the star in the course of its evolution ; there, it may be that $B$ has a high value in the wind, so that cosmic rays may reach energies in the $\mathrm{TeV}$ range. Other possibilities have been considered : oblique shocks, relativistic shocks, which are promising, but much more complicated to study, predicting spectra which are not automatically power laws. Another problem is that of the injection of particles in the shock acceleration, particularly in the case of electrons, which at subrelativistic energies have very small gyration radii, so that the waves that could scatter them are not there. It is very difficult to predict the ratio of electrons to protons arising from this mechanism - or in fact to understand how electrons are picked up at all.

And what about continuous acceleration throughout the cosmic ray lifetime, a la Fermi? In that scheme, on the average particles of higher energy have spent longer times in the galaxy, and thus traversed more matter. Thus, a model based solely on interstellar Fermi acceleration would predict that the grammage increases as $\ln \mathrm{E}$, and that the secondary/primary ratio increases with energy, contrary to observations.

However, a small amount of Fermi acceleration may take place in the interstellar medium. We could have a situation where cosmic rays are accelerated at the source (e.g. by a shock), and then suffer some reacceleration while they travel in the interstellar medium. The reacceleration may be due to the presence of large scale turbulence, or, in the case of a diffusion model, may be associated to the resonant interactions leading to 
pitch angle scattering and to space diffusion. Finally, particles may be reaccelerated through encounters with shock waves from other supernovae.

Now : between $10^{12}$ and $10^{14} \mathrm{eV}$, the cosmic ray anisotropy appears to be constant. If the cosmic ray escape time is decreasing when the energy increases, ( $t_{e} \alpha \lambda_{e} \alpha E^{-a}$ ), the anisotropy is also expected to increase with energy : $\left(\alpha \mathrm{E}^{\mathrm{a}}\right)$. But if weak reacceleration is introduced, it is possible to account for the observed primary/secondary ratio in the 5-20 $\mathrm{GeV} / \mathrm{n}$ range with a much slower dependence of $\lambda_{\mathrm{e}}$ with energy, thus predicting a much flatter anisotropy variation. However, models without reacceleration tend to predict

somewhat lower secondary to primary ratios at energies higher than $\approx 15 \mathrm{GeV} / \mathrm{n}$. Some data is available at these energies - particularly the recent results by Swordy et al. (1990), using transition radiation detectors - but the errors are still too large to distinguish between the various schemes.

Reacceleration by encounters with shocks from various supernovae has been considered by various authors, and more especially by Blandford and Ostriker (1980) (see review by Cesarsky, 1988). In this scenario, the secondary to primary ratio becomes constant at high energy, and is already too high compared with observations at $15 \mathrm{GeV} / \mathrm{n}$. In fact, the observed secondary/primary ratios, together with the diffusive shock acceleration mechanism, lead to results inconsistent with observations if standard assumption are used :

i) one supernova every 30 years in the galaxy

ii) a uniform galactic cosmic ray density

iii) most of the interstellar medium filled with hot gas $\left(\mathrm{n}=10^{-3} \mathrm{~cm}^{-3}, \mathrm{~T}=10^{6} \mathrm{~K}\right)$.

So : either i) the interstellar medium at large is much denser, or ii) supernova are not distributed uniformly, but they tend to explode in dense regions of the i s $\mathrm{m}$, or iii) cosmic rays are confined to small volumes within the galaxy, and we are sampling, with "our" cosmic rays, a small region of the is $\mathrm{m}$.

Up to now we have discussed secondaries of heavy nuclei. In fact, protons also travel in the interstellar medium, undergo collisions and generate their own secondaries

positrons and antiprotons. It appears that, at energy $\approx 10 \mathrm{GeV}$, antiprotons are about 6 times more numerous than predicted by the leaky-box model. The simplest way to interpret these observations (Lagage and Cesarsky, 1985 and reference therein) is to say that, in addition to the usual cosmic ray sources, "thick" sources are also present in the galaxy. These are supernovae or stellar winds embedded in a dense cloud, emitting cosmic rays which have to traverse a grammage $\lambda_{\mathrm{s}}$ before escaping to the is $\mathrm{m}$. If $\lambda_{\mathrm{s}}$ $>30 \mathrm{~g} / \mathrm{cm}^{2}$, essentially all the nuclei are broken by spallation reactions, and only protons and antiprotons emerge, plus of course secondary electrons and positrons and neutrinos. The antiproton observations are explained if $25 \%$ of the protons come from thick sources with $\lambda_{s}=30 \mathrm{~g} / \mathrm{cm}^{2}$. As for the positrons, results have been obtained by Müller and Tang (1990). The ratio $\mathrm{e}^{+} / \mathrm{e}^{+}+\mathrm{e}^{-}$is very high around $0.1 \mathrm{GeV}$, approaching 0.5 , this may imply that at these energies most electrons are secondary, produced in thick sources.

Is there a galactic wind powered by cosmic rays ? Breitschwerdt et al. $(1987,1990)$ considered the flow along a magnetic flux tube perpendicular to the galactic disk. They showed that this flow can be depicted as a de Laval nozzle, where the flow becomes 
supersonic at a height of $\approx 35 \mathrm{kpc}$. At the base (height $1 \mathrm{Kpc}$ ) the gas outflow velocity is $10 \mathrm{Km} / \mathrm{s}$; at $100 \mathrm{Kpc}$, it becomes $240 \mathrm{Km} / \mathrm{s}$; the total galactic mass loss rate is 0.33

$\mathrm{M}_{\mathrm{o} / \mathrm{yr}}$. Now : the change of slope of $\lambda_{\mathrm{e}}$ at $\approx 5 \mathrm{GV}$ is often interpreted as a passage from energy dependent diffusion to energy independent convection (Jones, 1991 and references therein). The corresponding escape velocity of cosmic ray in this "dynamic halo" model is in fact slow : 8 to $20 \mathrm{~km} / \mathrm{s}$, much less than the Alfven velocity $(=60 \mathrm{~km} / \mathrm{s})$. And one in fact expects that the cosmic ray velocity be the sum of $v_{A}+v_{\text {gas }}$, if the cosmic rays are strongly coupled to a galactic wind. Note that this model can be reconciled with the galactic wind model of Breitschwerdt et al. if the galactic field is mostly parallel to the disk, but if vertical flux tubes are also present over a fraction ( 8 $\mathrm{Km} / \mathrm{s}) /\left(\mathrm{v}_{\text {gas }}+\mathrm{v}_{\mathrm{A}}\right)=10 \%$ of the surface at height $1 \mathrm{Kpc}$.

High energy gamma rays ( $>30 \mathrm{MeV}$ ) are another type of cosmic ray secondary : they are generated by collisions of cosmic ray nuclei ( $\pi^{\circ}$ decay) or electrons (bremsstrahlung) with interstellar medium particles. The galactic distribution of cosmic rays, as measured by SAS 2 and in much more detail by COS-B, has been compared to the $\mathrm{HI}$ and $\mathrm{CO}$ data by many authors. A detailed fit by Strong et al. (1988) leads to a shallow gradient of cosmic rays with galactocentric distance in the high energy range (dominated by $\pi^{\circ}$ gamma rays), and a steeper gradient in the low energy range (dominated by bremsstrahlung). This difference in slope can be interpreted as revealing the presence of secondary electrons partially trapped in molecular clouds, as expected if thick sources are present. The shallow gradient in the primary component disagrees with all the existing diffusion models, even those featuring a large halo. A better understanding of the coupling between matter and cosmic rays at large and small scales should come from the observations of EGRET, aboard the Compton Observatory.

Over the last few years, the existence of a very tight correlation between radio synchrotron and infrared radiation has been established, at various scales in the universe (e.g. Helou 1990 and references there in). The simplest explanation is that both cosmic ray electrons and UV photons, later absorbed to produce infrared photons, all originate mostly in star forming regions, where massive stars become supernovae. The efficiency of electron acceleration is then proportional to the UV luminosity at early times, and the scatter in the diagram just reflects the scatter in magnetic field strengths.

Bicay and Helou (1990) note that in spiral galaxies, the ratio of infrared to radio emission decreases away from the nuclei. This may reflect the broadening of the cosmic ray distribution with respect to the source distribution, due to propagation effects. It is interesting that secondary electrons have been disregarded in all interpretations.

With ROSAT, GRANAT and COMPTON, we are entering again in an exciting area in high energy astrophysics. This will be highlighted at another joint discussion in this General Assembly. I just mention a few intriguing results, well deserving further investigation :

a) the nature of the galactic $X$ ray ridge, observed by various satellites, and particularly by Ginga (Koyama et al. 1989) is not yet understood. Its spectrum in the range 3-10 $\mathrm{KeV}$ is that of a hot, thin plasma, and the iron line is present everywhere. There is an important, broad maximum in the central regions of the galaxy (Yamauchi et al, 1990). Despite its much lower energy range, ROSAT may help in deciding if this emission is really extended or due to the superposition of numerous point sources.

b) Extended gamma ray sources start to be apparent in the COS-B data. For instance, an excess may be present in the Cepheus flare (Grenier et al. 1989, Grenier and Lebrun 
1990), correlated with two non thermal radio spurs and with an excess in soft $X$ rays. This may be a bubble of hot gas between two clouds.

c) ROSAT has discovered a new supernova remnant, which in $\mathrm{X}$ rays is one of the ten brightest such objects in the galaxy (Pfeffermann et al. 1991). In the radio range, it is quite faint, and had not been detected. Will ROSAT change our supernova statistics?

d) Finally, an intriguing ROSAT result : the apparent shadowing of a halo emission at $0.25 \mathrm{KeV}$ by the high latitude Draco cloud, several hundred parsecs away. If this interpretation is confirmed, it will be the first indication of the presence of an $\mathrm{X}$ ray halo around our galaxy (Burrows and Mendenhall 1991, but see also Snowden et al., 1991)

\section{References}

Bicay, M.D. and Helou, G. 1990, Astrophys. J. 362, 59

Blandford, R.D., et Eichler, D., 1987, Phys. Report 154, 1.

Blandford, R.D. and Ostriker, J.P., 1980, Astrophys. J. 237, 793

Breitschwerdt, Mc Kenzie and Völk, 1987,20th Int. Cosmic Ray Conf. 2, 115 and 1990, 21st Int. Cosmic Ray Conf. 3, 315

Burnett, T.H., et al. 1990, Astrophys. J. Letters

Burrows, P.H. and Mendenhall, J.A., 1991, Nature, 351, 629

Cesarsky, C.J. 1988, 20th Int. Cosmic Ray Conference, 8, 87

Engelmann, J.J., et al., 1990, Astron. Astrophys. 233, 96

Fermi, E., 1954, Astrophys. J. 119, 1

Ginzburg, V.L., Khazan, Y.M., Ptuskin, V.S., 1980, Astrophys. Space Sci. 68, 295

Grenier, I.A. and Lebrun, F., 1990, Astrophys. J. 360, 129

Grenier, I.A., et al., 1989, Astrophys. J. 347, 231

Helou, G., 1990, in "The interpretation of Modern synthesis observation of spiral galaxies", Albuquerque Workshop, in press

Jones, F., 1991, 144th IAU Symp., p. 359

Koyama, 1989, Publ. Astron. Soc. Japan, 41, 665

Lagage, P.O. and Cesarsky, C.J., 1983, Astron. Astrophys. 125, 249

Lagage, P.O. and Cesarsky, C.J., 1985, Astron. Astrophys. 147, 127

Müller, D. and Tang, K.K., 1990, 21 st Int. Cosmic Ray Conf., 3, 249

Parker, E.N., 1969, Space Sci. Rev. 9, 651

Pfeffermann, E., Aschenbach, B. and Predehl, P., 1991, Astron. Astrophys. L.28

Snowder, S.L., et al., 1991, Science, 252, 1529

Strong, A., et al. 1988, Astron. Astrophys. 207, 1

Swordy, S.P. et al., 1990, Astrophys. J. 349, 625

Völk, H. and Biermann, P., 1988, Astrophys. J. Letters 333, L65

Yamauchi, S. et al., 1990, Astrophys. J. 365, 532 